\title{
Pathwise estimation of stochastic functional Kolmogorov-type systems with infinite delay
}

Enwen Zhu ${ }^{1}$ and Yong $\mathrm{Xu}^{2^{*}}$

${ }^{*}$ Correspondence:
xuyonghust@126.com
${ }^{2}$ School of Mathematics, Central
South University, Changsha, Hunan
410075, China
Full list of author information is
available at the end of the article

available at the end of the article

\section{Springer}

\begin{abstract}
In this paper, we study pathwise estimation of the global positive solutions for the stochastic functional Kolmogorov-type systems with infinite delay. Under some conditions, the growth rate of the solutions for such systems with general noise structures is less than a polynomial rate in the almost sure sense. To illustrate the applications of our theory more clearly, this paper also discusses various stochastic Lotka-Volterra-type systems as special cases.
\end{abstract}

MSC: $34 \mathrm{~K} 50 ; 60 \mathrm{H} 10 ;$ 92D25; 93E03

Keywords: stochastic functional Kolmogorov systems; Lotka-Volterra systems; pathwise estimation; infinite delay

\section{Introduction}

The stochastic functional Kolomogorov-type systems for $n$ interacting species is described by the following stochastic functional differential equation:

$$
\mathrm{d} x(t)=\operatorname{diag}\left(x_{1}, \ldots, x_{n}\right)\left[f\left(x_{t}\right) \mathrm{d} t+g\left(x_{t}\right) \mathrm{d} w(t)\right]
$$

where $x=\left(x_{1}, \ldots, x_{n}\right)^{T}, \operatorname{diag}\left(x_{1}, \ldots, x_{n}\right)$ represents the $n \times n$ matrix with all elements zero except those on the diagonal which are $x_{1}, \ldots, x_{n}, w(t)$ is a scalar Brownian motion, and

$$
f=\left(f_{1}, \ldots, f_{n}\right)^{T}: C\left([-\tau, 0] ; \mathbb{R}^{n}\right) \rightarrow \mathbb{R}^{n}, \quad g=\left(g_{1}, \ldots, g_{n}\right)^{T}: C\left([-\tau, 0] ; \mathbb{R}^{n}\right) \rightarrow \mathbb{R}^{n}
$$

There is an extensive literature concerned with the dynamics of this system and we here only mention $[7,9,10]$. References $[7,9,10]$ study existence and uniqueness of the global positive solution of Eq. (1.1), and its asymptotic bound properties and moment average in time. The nice positive property provides us with a great opportunity to discuss further how the solutions vary in $\mathbb{R}_{+}^{n}$ in more detail. Our interest is to discuss pathwise estimation of the global positive solutions for stochastic functional Kolomogorov-type systems with infinite delay.

There is an extensive literature concerned with the dynamics of the Kolomogorov-type systems (1.1) without the stochastic perturbation and we here only mention [3-5, 21]. As a special case, multispecies Lotka-Volterra-type systems with bounded delay and unbounded delay are studied. For example, He and Gopalsamy [16] consider the global positive solution for a two-dimensional Lotka-Volterra system. Kuang [19] examines global 
stability for infinite delay Lotka-Volterra-type systems. For more details about the LotkaVolterra-type systems, we refer the reader to see $[6,11-13,15,20]$ and references therein. In fact, population systems are often subject to environmental noise. It is therefore useful to reveal how the noise affects on the Kolmogorov-type systems. Recently, the stochastic Lotka-Volterra systems have received increasing attention. References $[1,17]$ reveal that the noise plays an important role to suppress the growth of the solution. References [2,18] show the stochastic system behaves similarly to the corresponding deterministic system under different stochastic perturbations, respectively. These indicate clearly that different structures of environmental noise may have different effects on Lotka-Volterra systems.

However, little is yet known about the pathwise property of the stochastic functional Kolmogorov-type systems with infinite delay although they may be seen as a generalized stochastic functional Lotka-Volterra system. This paper will examine the pathwise estimation of the stochastic functional Kolmogorov-type systems under the general noise structures. Consider the $n$-dimensional unbounded delay stochastic functional Kolmogorovtype systems

$$
\mathrm{d} x(t)=\operatorname{diag}\left(x_{1}(t), \ldots, x_{n}(t)\right)\left[f\left(x_{t}\right) \mathrm{d} t+g\left(x_{t}\right) \mathrm{d} w(t)\right]
$$

on $t \geq 0$, where $w(t)$ is an $m$-dimensional Brownian motion, and

$$
f: B C\left((-\infty, 0] ; \mathbb{R}^{n}\right) \rightarrow \mathbb{R}^{n}, \quad g: B C\left((-\infty, 0] ; \mathbb{R}^{n}\right) \rightarrow \mathbb{R}^{n \times m},
$$

where the initial data space $B C\left((-\infty, 0] ; \mathbb{R}^{n}\right)$ denotes the family of bounded continuous $\mathbb{R}^{n}$-value functions $\varphi$ defined on $(-\infty, 0]$ with the norm $\|\varphi\|=\sup _{\theta \leq 0}|\varphi(\theta)|<\infty$. Here, $f$ and $g$ are local Lipschitz continuous. Clearly, Eq. (1.2) includes the following forms:

$$
\begin{aligned}
& \mathrm{d} x(t)=\operatorname{diag}\left(x_{1}(t), \ldots, x_{n}(t)\right)\left[f\left(x_{t}\right) \mathrm{d} t+g(x(t)) \mathrm{d} w(t)\right], \\
& \mathrm{d} x(t)=\operatorname{diag}\left(x_{1}(t), \ldots, x_{n}(t)\right)\left[f(x(t)) \mathrm{d} t+g\left(x_{t}\right) \mathrm{d} w(t)\right] .
\end{aligned}
$$

Since this paper mainly examines the pathwise estimation of the solution for stochastic functional Kolmogorov-type systems with infinite delay, we assume that there exists a unique global positive solution for all discussed equations (see $[8,14]$ ).

In the next section, we give some necessary notations and lemmas. To show our idea clearly, Section 2 also studies the pathwise estimation for general stochastic functional differential equations with infinite delay. Applying the result of Section 2, we give various conditions under which stochastic functional Kolmogorov systems with infinite delay show the nice pathwise properties in Section 3. As in the applications of Section 2 and Section 3, Section 4 discusses several special equations, including various stochastic Lotka-Volterra systems with infinite delay.

\section{A general result}

Throughout this paper, unless otherwise specified, we use the following notations. Let $|\cdot|$ denote the Euclidean norm in $\mathbb{R}^{n}$. If $A$ is a vector or matrix, its transpose is denoted by $A^{T}$. If $A$ is matrix, its trace norm is denoted by $|A|=\sqrt{\operatorname{trace}\left(A^{T} A\right)}$. Let $\mathbb{R}_{-}^{n}=\left\{x \in \mathbb{R}^{n}: x_{i} \leq\right.$ 0 for all $1 \leq i \leq n\}, \mathbb{R}_{+}^{n}=\left\{x \in \mathbb{R}^{n}: x_{i} \geq 0\right.$ for all $\left.1 \leq i \leq n\right\}, \mathbb{R}_{++}^{n}=\left\{x \in \mathbb{R}^{n}: x_{i}>0\right.$ for all $1 \leq$ $i \leq n\}$. For any $c=\left(c_{1}, \ldots, c_{n}\right)^{T} \in \mathbb{R}_{++}^{n}$, let $\hat{c}=\min \left\{c_{1}, \ldots, c_{n}\right\}$ and $\check{c}=\max \left\{c_{1}, \ldots, c_{n}\right\}$. Let 
$(\Omega, \mathcal{F}, \mathbb{P})$ be a complete probability space with a filtration $\left\{\mathcal{F}_{t}\right\}_{t \geq 0}$ satisfying the usual conditions, that is, it is right continuous and increasing while $\mathcal{F}_{0}$ contains all $P$-null sets. Let $w(t)$ be an $m$-dimensional Brownian motion defined on the complete probability space. If $x(t)$ is an $\mathbb{R}^{n}$-valued stochastic process on $t \in \mathbb{R}$, we let $x_{t}=\{x(t+\theta): \theta \in(-\infty, 0]\}$ for $t \geq 0$.

Let $\mathcal{M}_{0}$ be the family of the probability measures $\mu$ on $(-\infty, 0]$. Let $\varepsilon_{0}>0$. For any $\varepsilon \in\left[0, \varepsilon_{0}\right]$, we define a set of the probability measure

$$
\mathcal{M}_{\varepsilon}:=\left\{\mu \in \mathcal{M}_{0}, \mu_{\varepsilon}:=\int_{-\infty}^{0} e^{-\varepsilon \theta} \mathrm{d} \mu(\theta)<\infty\right\} .
$$

Clearly, $\mathcal{M}_{\varepsilon_{0}} \subseteq \mathcal{M}_{\varepsilon} \subseteq \mathcal{M}_{0}$ and $\mu_{\varepsilon}$ is continuously dependent on $\varepsilon$ on $\left[0, \varepsilon_{0}\right]$, and $\mu_{\varepsilon} \rightarrow 1$ as $\varepsilon \rightarrow 0$. For the arbitrary $V(x) \in C^{2}\left(\mathbb{R}^{n} ; \mathbb{R}\right)$, define

$$
V_{x}(x)=\left(\frac{\partial V(x)}{\partial x_{1}}, \ldots, \frac{\partial V(x)}{\partial x_{n}}\right), \quad V_{x x}(x)=\left(\frac{\partial^{2} V(x)}{\partial x_{i} x_{j}}\right)_{n \times n}
$$

The following lemma shows boundedness of polynomial functions.

Lemma 2.1 For any positive constants $b, \alpha$, and the function $h(x) \in C\left(\mathbb{R}_{+}^{n} ; \mathbb{R}\right)$, if $h(x)=$ $o\left(|x|^{\alpha}\right)$, as $|x| \rightarrow \infty$. Then

$$
\sup _{x \in \mathbb{R}_{+}^{n}}\left[-b|x|^{\alpha}+h(x)\right]<\infty
$$

Proof We may choose $c>0$ such that $|h(x)|<|x|^{\alpha}$ for any $|x|^{\alpha}>c$, which implies $-b|x|^{\alpha}+$ $h(x)<0$. Therefore, we have

$$
\sup _{x \in \mathbb{R}_{+}^{n}}\left[-b|x|^{\alpha}+h(x)\right]=\sup _{x \in \mathbb{R}_{+}^{n},|x|<c}\left[-b|x|^{\alpha}+h(x)\right]<\infty,
$$

as required.

To show our idea about the pathwise estimation of solutions clearly, we first consider the following general $n$-dimensional stochastic functional differential equation:

$$
\mathrm{d} x(t)=F\left(t, x_{t}\right) \mathrm{d} t+G\left(t, x_{t}\right) \mathrm{d} w(t)
$$

on $t \geq 0$. Here,

$$
F: \mathbb{R}_{+} \times B C\left(\mathbb{R}_{-} ; \mathbb{R}^{n}\right) \rightarrow \mathbb{R}^{n}, \quad G: \mathbb{R}_{+} \times B C\left(\mathbb{R}_{-} ; \mathbb{R}^{n}\right) \rightarrow \mathbb{R}^{n \times m}
$$

are local Lipschitz continuous and $w(t)$ is an $m$-dimensional Brownian motion. Assume that Eq. (2.1) almost surely admits a unique global positive solution. Then we have the following pathwise estimation.

Theorem 2.1 Let $p>0$ and $x(t)=x(t, \xi)$ be the global positive solution of Eq. (2.1). If there exists a vector $c \in \mathbb{R}_{++}^{n}$ such that for any $\varepsilon \in\left(0, \varepsilon_{0}\right]$ and an integer $J>0$, there are constants 
$K, K_{j}>0, \alpha_{j}$ and probability measures $\mu_{j} \in \mathcal{M}_{\varepsilon}(1 \leq j \leq J)$ such that

$$
\begin{aligned}
& \frac{c^{T} F(t, \varphi)}{c^{T} \varphi(0)}+\frac{p-1}{2}\left[\frac{c^{T} G(t, \varphi)}{c^{T} \varphi(0)}\right]^{2}+\varepsilon \log \left(c^{T} \varphi(0)\right) \\
& \leq K+\sum_{j=1}^{J} K_{j}\left(\int_{-\infty}^{0}|\varphi(\theta)|^{\alpha_{j}} \mathrm{~d} \mu_{j}(\theta)-\mu_{j \varepsilon}|\varphi(0)|^{\alpha_{j}}\right)
\end{aligned}
$$

for any $t \geq 0$ and $\varphi \in B C\left(\mathbb{R}_{-} ; \mathbb{R}_{++}^{n}\right)$. Then for any given initial data $\xi \in B C\left(\mathbb{R}_{-} ; \mathbb{R}_{++}^{n}\right) \cap$ $L^{q}\left(\mathbb{R}_{-} ; \mathbb{R}^{n}\right)$, where $q=\min _{1 \leq j \leq J}\left(\alpha_{j}\right)$, the solution $x(t)$ of $E q$. (2.1) has the following property:

$$
\limsup _{t \rightarrow \infty} \frac{\log (|x(t)|)}{\log t} \leq \frac{1}{p} \quad \text { a.s. }
$$

Proof Define

$$
V(x)=c^{T} x, \quad \text { on } x \in \mathbb{R}_{+}^{n} .
$$

For any $\varepsilon \in\left(0, \varepsilon_{0}\right]$, applying the Itô formula to $e^{\varepsilon t} \log V(x(t))$ yields

$$
\begin{aligned}
e^{\varepsilon t} & \log V(x(t)) \\
= & \log V(x(0))+\int_{0}^{t} \mathrm{~d}\left[e^{\varepsilon s} \log V(x(s))\right] \\
= & \log V(\xi(0)) \\
& +\int_{0}^{t} e^{\varepsilon s}\left[\frac{c^{T} F\left(s, x_{s}\right)}{c^{T} x(s)}-\frac{1}{2}\left(\frac{c^{T} G\left(s, x_{s}\right)}{c^{T} x(s)}\right)^{2}+\varepsilon \log V(x(s))\right] \mathrm{d} s+M(t),
\end{aligned}
$$

where

$$
M(t)=\int_{0}^{t} e^{\varepsilon s} \frac{c^{T} G\left(s, x_{s}\right)}{c^{T} x(s)} \mathrm{d} w(s)
$$

is a continuous local martingale with the quadratic variation

$$
\langle M(t), M(t)\rangle=\int_{0}^{t} e^{2 \varepsilon s}\left(\frac{c^{T} G\left(s, x_{s}\right)}{c^{T} x(s)}\right)^{2} \mathrm{~d} s .
$$

For any given $k \in \mathbb{N}$ and $\delta>1$, the exponential martingale inequality yields

$$
\mathbb{P}\left\{\sup _{0 \leq t \leq k}\left[M(t)-\frac{p}{2 e^{\varepsilon k}} \int_{0}^{t} e^{2 \varepsilon s}\left(\frac{c^{T} G\left(s, x_{s}\right)}{c^{T} x(s)}\right)^{2} \mathrm{~d} s\right] \geq \frac{\delta e^{\varepsilon k} \log n}{p}\right\} \leq \frac{1}{k^{\delta}} .
$$

Since $\Sigma_{k=1}^{\infty} n^{-\delta}<\infty$, the well-known Borel-Cantelli lemma yields that there exists an $\Omega_{0} \subset$ $\Omega$ with $\mathbb{P}\left(\Omega_{0}\right)=1$ such that for any $\omega \in \Omega_{0}$ there exists an integer $k_{0}(\omega)$, when $k \geq k_{0}(\omega)$ and $k-1 \leq t \leq k$,

$$
M(t) \leq \frac{p}{2} \int_{0}^{t} e^{\varepsilon s}\left(\frac{c^{T} G\left(s, x_{s}\right)}{c^{T} x(s)}\right)^{2} \mathrm{~d} s+\frac{\delta e^{\varepsilon(t+1)} \log (t+1)}{p} .
$$


Zhu and Xu Journal of Inequalities and Applications 2012, 2012:171

Page 5 of 15

http://www.journalofinequalitiesandapplications.com/content/2012/1/171

Substituting the inequalities (2.5) into (2.4), and letting $t$ sufficiently large, it is obtained almost surely that

$$
e^{\varepsilon t} \log V(x(t)) \leq \log V(\xi(0))+p^{-1} \delta e^{\varepsilon(t+1)} \log (t+1)+I,
$$

where

$$
I=\int_{0}^{t} e^{\varepsilon s}\left[\frac{c^{T} F\left(s, x_{s}\right)}{c^{T} x(s)}+\frac{p-1}{2}\left(\frac{c^{T} G\left(s, x_{s}\right)}{c^{T} x(s)}\right)^{2}+\varepsilon \log V(x(s))\right] \mathrm{d} s .
$$

By the condition (2.2), we obtain that

$$
\begin{aligned}
I & \leq \int_{0}^{t} e^{\varepsilon s}\left\{K+\sum_{j=1}^{J} K_{j}\left[\int_{-\infty}^{0}|x(s+\theta)|^{\alpha_{j}} \mathrm{~d} \mu_{j}(\theta)-\mu_{j \varepsilon}|x(s)|^{\alpha_{j}}\right]\right\} \mathrm{d} s \\
& \leq \varepsilon^{-1} K e^{\varepsilon t}+\sum_{j=1}^{J} K_{j} \int_{0}^{t} e^{\varepsilon s}\left(\int_{-\infty}^{0}|x(s+\theta)|^{\alpha_{j}} \mathrm{~d} \mu_{j}(\theta)-\mu_{j \varepsilon}|x(s)|^{\alpha_{j}}\right) \mathrm{d} s .
\end{aligned}
$$

We may compute that

$$
\begin{aligned}
& \int_{0}^{t} e^{\varepsilon s} \int_{-\infty}^{0}|x(s+\theta)|^{\alpha_{j}} \mathrm{~d} \mu_{j}(\theta) \mathrm{d} s \\
& \quad=\int_{-\infty}^{0} \mathrm{~d} \mu_{j}(\theta) \int_{0}^{t} e^{\varepsilon s}|x(s+\theta)|^{\alpha_{j}} \mathrm{~d} s \\
& \quad=\int_{-\infty}^{0} \mathrm{~d} \mu_{j}(\theta) \int_{\theta}^{t+\theta} e^{\varepsilon(s-\theta)}|x(s)|^{\alpha_{j}} \mathrm{~d} s \\
& \quad \leq \int_{-\infty}^{0} e^{-\varepsilon \theta} \mathrm{d} \mu_{j}(\theta)\left[\int_{-\infty}^{0} e^{\varepsilon s}|x(s)|^{\alpha_{j}} \mathrm{~d} s+\int_{0}^{t} e^{\varepsilon s}|x(s)|^{\alpha_{j}} \mathrm{~d} s\right] \\
& \quad \leq \mu_{j \varepsilon} C_{\alpha_{j}}+\mu_{j \varepsilon} \int_{0}^{t} e^{\varepsilon s}|x(s)|^{\alpha_{j}} \mathrm{~d} s,
\end{aligned}
$$

where

$$
C_{\alpha_{j}}:=\int_{-\infty}^{0}|\xi(s)|^{\alpha_{j}} \mathrm{~d} s<\infty, \quad \mu_{j \varepsilon}:=\int_{-\infty}^{0} e^{-\varepsilon \theta} \mathrm{d} \mu_{j}(\theta)<\infty,
$$

for all $1 \leq j \leq J$. Therefore,

$$
e^{\varepsilon t} \log V(x(t)) \leq \log V(\xi(0))+\varepsilon^{-1} K e^{\varepsilon t}+\sum_{j=1}^{J} K_{j} \mu_{j \varepsilon} C_{\alpha_{j}}+p^{-1} \delta e^{\varepsilon(t+1)} \log (t+1) .
$$

This implies

$$
\limsup _{t \rightarrow \infty} \frac{\log (V(x(t)))}{\log t} \leq \frac{\delta e^{\varepsilon}}{p}, \quad \text { a.s. }
$$

Noting the inequality

$$
0<\hat{c}|x| \leq V(x) \leq|c||x|, \quad \text { for } x \in \mathbb{R}_{++}^{n},
$$


and letting $\delta \rightarrow 1, \varepsilon \rightarrow 0$, we obtain that

$$
\limsup _{t \rightarrow \infty} \frac{\log (|x(t)|)}{\log t} \leq \frac{1}{p}, \quad \text { a.s. }
$$

as required.

The result (2.3) shows that for any $\varepsilon>0$, there is a positive random time $T_{\varepsilon}$ such that, with probability one, for any $t>T_{\varepsilon}$,

$$
|x(t)|^{p} \leq t^{1+\varepsilon} .
$$

In other words, with probability one, the solution will not grow faster than $t^{(1+\varepsilon) / p}$.

In this theorem, it is a key to compute the condition (2.2). In the following section, we apply Theorem 2.1 to examine stochastic functional Kolmogorov-type systems.

\section{Main result}

This section mainly applies the result of Theorem 2.1 to Eq. (1.2). For any $x \in \mathbb{R}_{++}^{n}$ and $\varphi \in B C\left(\mathbb{R}_{-} ; \mathbb{R}_{++}^{n}\right)$, we firstly list the following conditions for both $f$ and $g$ that we will need.

(H1) These exist $\alpha>0, \kappa, \bar{\kappa} \geq 0$ and the probability measure $\mu \in \mathcal{M}_{\varepsilon}$, where $\varepsilon \in\left(0, \varepsilon_{0}\right]$ and $\alpha \leq \varepsilon$, such that

$$
|f(\varphi)| \leq \kappa|\varphi(0)|^{\alpha}+\bar{\kappa} \int_{-\infty}^{0}|\varphi(\theta)|^{\alpha} \mathrm{d} \mu(\theta)+\mathrm{o}\left(|\varphi(0)|^{\alpha}\right) .
$$

(H2) There exist $\beta>0, \lambda, \bar{\lambda} \geq 0$ and the probability measure $v \in \mathcal{M}_{\varepsilon}$, where $\varepsilon \in\left(0, \varepsilon_{0}\right]$ and $\beta \leq \varepsilon$, such that

$$
|g(\varphi)| \leq \lambda|\varphi(0)|^{\beta}+\bar{\lambda} \int_{-\infty}^{0}|\varphi(\theta)|^{\beta} \mathrm{d} v(\theta)+\mathrm{o}\left(|\varphi(0)|^{\beta}\right) .
$$

(H3) There exist $c=\left(c_{1}, \ldots, c_{n}\right)^{T} \in \mathbb{R}_{++}^{n}, \beta, b>0, \sigma \geq 0$ and the probability measure $\bar{v} \in$ $\mathcal{M}_{\varepsilon}$, where $\varepsilon \in\left(0, \varepsilon_{0}\right]$ and $2 \beta \leq \varepsilon$, such that

$$
|\varphi(0)|^{-2}\left[\varphi^{T}(0) C g(\varphi)\right]^{2} \geq b|\varphi(0)|^{2 \beta}-\sigma \int_{-\infty}^{0}|\varphi(\theta)|^{2 \beta} \mathrm{d} \bar{\nu}(\theta)+\mathrm{o}\left(|\varphi(0)|^{2 \beta}\right),
$$

where $C=\operatorname{diag}\left(c_{1}, \ldots, c_{n}\right)$.

(H4) There exist $c \in \mathbb{R}_{++}^{n}, \alpha, b>0, \sigma \geq 0$ and the probability measure $\bar{\mu} \in \mathcal{M}_{\varepsilon}$, where $\varepsilon \in$ $\left(0, \varepsilon_{0}\right]$ and $\alpha \leq \varepsilon$, such that

$$
|\varphi(0)|^{-1} \varphi^{T}(0) C f(\varphi) \leq-b|\varphi(0)|^{\alpha}+\sigma \int_{-\infty}^{0}|\varphi(\theta)|^{\alpha} \mathrm{d} \bar{\mu}(\theta)+\mathrm{o}\left(|\varphi(0)|^{\alpha}\right),
$$

where $C=\operatorname{diag}\left(c_{1}, \ldots, c_{n}\right)$.

When $f(x)$ and $g(x)$ replace $f(\varphi)$ and $g(\varphi)$, the above conditions may be rewritten as

$\left(\mathrm{H} 1^{\prime}\right)$ These exist $\alpha>0, \kappa \geq 0$ such that

$$
|f(x)| \leq \kappa|x|^{\alpha}+\mathrm{o}\left(|x|^{\alpha}\right)
$$


$\left(\mathrm{H} 2^{\prime}\right)$ There exist $\beta>0, \lambda \geq 0$ such that

$$
|g(x)| \leq \lambda|x|^{\beta}+\mathrm{o}\left(|x|^{\beta}\right)
$$

$\left(\mathrm{H}^{\prime}\right)$ There exist $c \in \mathbb{R}_{++}^{n}, \beta, b>0$ such that

$$
\left[x^{T} C g(x)\right]^{2} \geq b|x|^{2 \beta+2}+\mathrm{o}\left(|x|^{2 \beta+2}\right)
$$

where $C=\operatorname{diag}\left(c_{1}, \ldots, c_{n}\right)$.

$\left(\mathrm{H} 4^{\prime}\right)$ There exist $c \in \mathbb{R}_{++}^{n}, \alpha, b>0$ such that

$$
x^{T} C f(x) \leq-b|x|^{\alpha+1}+\mathrm{o}\left(|x|^{\alpha+1}\right)
$$

where $C=\operatorname{diag}\left(c_{1}, \ldots, c_{n}\right)$.

Here, we emphasize that the same letter represents the same parameter when we use the several conditions simultaneously. In the following sections, we always assume that the initial data $x_{0} \in B C\left(\mathbb{R}_{-}^{n} ; \mathbb{R}_{++}^{n}\right) \cap L^{(\alpha \wedge \beta)}\left(\mathbb{R}_{-}^{n} ; \mathbb{R}^{n}\right)$. Applying the conditions (H1) and (H3) to stochastic functional Kolmogorov systems (1.2) gives

Theorem 3.1 Under the conditions (H1) and (H3), for the global positive solution $x(t)$ of Eq. (1.2), if one of the following conditions

$$
\begin{aligned}
& p=1, \quad \alpha<2 \beta, \quad b>\sigma ; \\
& 0<p<1, \quad \alpha=2 \beta, \quad b>\sigma+\frac{2|c|^{2}(\kappa+\bar{\kappa})}{1-p}
\end{aligned}
$$

holds, then

$$
\limsup _{t \rightarrow \infty} \frac{\log \left(|x(t)|^{p}\right)}{\log (t)} \leq 1, \quad \text { a.s. }
$$

Proof To apply Theorem 2.1, we need to compute the condition (2.2). Substituting $F(t, \varphi)=\operatorname{diag}\left(\varphi_{1}(0), \ldots, \varphi_{n}(0)\right) f(\varphi)$ and $G(t, \varphi)=\operatorname{diag}\left(\varphi_{1}(0), \ldots, \varphi_{n}(0)\right) g(\varphi)$ into the condition (2.2) yields

$$
\begin{aligned}
\Phi_{\varepsilon} & :=\frac{\varphi^{T}(0) C f(\varphi)}{c^{T} \varphi(0)}+\frac{p-1}{2}\left[\frac{\varphi^{T}(0) C g(t, \varphi)}{c^{T} \varphi(0)}\right]^{2}+\varepsilon \log \left(c^{T} \varphi(0)\right) \\
& =: I_{1}+I_{2}+\varepsilon \log \left(c^{T} \varphi(0)\right),
\end{aligned}
$$

where $C=\operatorname{diag}\left(c_{1}, \ldots, c_{n}\right)$. By the condition $(\mathrm{H} 1)$,

$$
\begin{aligned}
I_{1} & \leq \max _{1 \leq i \leq n}\left\{f_{i}(\varphi)\right\} \\
& \leq|f(\varphi)| \\
& \leq \kappa|\varphi(0)|^{\alpha}+\bar{\kappa} \int_{-\infty}^{0}|\varphi(\theta)|^{\alpha} \mathrm{d} \mu(\theta)+\mathrm{o}\left(|\varphi(0)|^{\alpha}\right) .
\end{aligned}
$$


Noting $p \leq 1$, by the condition (H3),

$$
\begin{aligned}
I_{2} & \leq \frac{p-1}{2|c|^{2}|\varphi(0)|^{2}}\left[\varphi^{T} C g(\varphi)\right]^{2} \\
& \leq \frac{p-1}{2|c|^{2}}\left[b|\varphi(0)|^{2 \beta}-\sigma \int_{-\infty}^{0}|\varphi(\theta)|^{2 \beta} \mathrm{d} \bar{v}(\theta)+\mathrm{o}\left(|\varphi(0)|^{2 \beta}\right)\right] .
\end{aligned}
$$

Substituting $I_{1}$ and $I_{2}$ into (3.3) and noting that $\varepsilon \log \left(c^{T} \varphi(0)\right) \leq \mathrm{o}\left(|\varphi(0)|^{\alpha}\right)$, we get

$$
\begin{aligned}
\Phi_{\varepsilon} \leq & \bar{\kappa} \int_{-\infty}^{0}|\varphi(\theta)|^{\alpha} \mathrm{d} \mu(\theta)+\frac{\sigma(1-p)}{2|c|^{2}} \int_{-\infty}^{0}|\varphi(\theta)|^{2 \beta} \mathrm{d} \bar{\nu}(\theta) \\
& +\kappa|\varphi(0)|^{\alpha}-\frac{b(1-p)}{2|c|^{2}}|\varphi(0)|^{2 \beta}+\mathrm{o}\left(|\varphi(0)|^{2 \beta}\right) \\
= & \bar{\kappa}\left[\int_{-\infty}^{0}|\varphi(\theta)|^{\alpha} \mathrm{d} \mu(\theta)-\mu_{\varepsilon}|\varphi(0)|^{\alpha}\right] \\
& +\frac{\sigma(1-p)}{2|c|^{2}}\left[\int_{-\infty}^{0}|\varphi(\theta)|^{2 \beta} \mathrm{d} \bar{v}(\theta)-\bar{v}_{\varepsilon}|\varphi(0)|^{2 \beta}\right]+I,
\end{aligned}
$$

where

$$
I=-\frac{1-p}{2|c|^{2}}\left(b-\sigma \bar{v}_{\varepsilon}\right)|\varphi(0)|^{2 \beta}+\left(\kappa+\bar{\kappa} \mu_{\varepsilon}\right)|\varphi(0)|^{\alpha}+o\left(|\varphi(0)|^{2 \beta}\right) .
$$

If the condition (3.1a) holds, choosing $\delta \in(0,1)$ to replace $p$ in (3.4), then we have

$$
I=-\frac{1-\delta}{2|c|^{2}}\left(b-\sigma \bar{v}_{\varepsilon}\right)|\varphi(0)|^{2 \beta}+\mathrm{o}\left(|\varphi(0)|^{2 \beta}\right)=:-b_{1}|\varphi(0)|^{2 \beta}+\mathrm{o}\left(|\varphi(0)|^{2 \beta}\right) \text {. }
$$

Noting $b>\sigma$ and $\bar{\nu}_{\varepsilon} \rightarrow 1$ as $\varepsilon \rightarrow 0$, choose $\varepsilon$ sufficiently small such that $b_{1}>0$. Lemma 2.1 gives $I \leq$ const, which implies the condition (2.2) is satisfied. Letting $\delta \rightarrow 1$, Theorem 2.1 gives the desired result under $p=1$.

If the condition (3.1b) holds,

$$
\begin{aligned}
I & =-\frac{1-p}{2|c|^{2}}\left(b-\sigma \bar{\nu}_{\varepsilon}-\frac{2|c|^{2}\left(\kappa+\bar{\kappa} \mu_{\varepsilon}\right)}{1-p}\right)|\varphi(0)|^{2 \beta}+o\left(|\varphi(0)|^{2 \beta}\right) \\
& =:-b_{2}|\varphi(0)|^{2 \beta}+o\left(|\varphi(0)|^{2 \beta}\right) .
\end{aligned}
$$

By the condition (3.1b), choose $\varepsilon$ sufficiently small such that $b_{2}>0$, so we have $I \leq$ const, which implies the condition (2.2) and further gives the desired result by Theorem 2.1.

Applying Theorem 3.1 to Eq. (1.3), the condition (H3) should be replaced by $\left(\mathrm{H}^{\prime}\right)$, which implies $\sigma=0$. This result may be described as follows.

Corollary 3.2 Under the conditions (H1) and (H3'), for the global positive solution $x(t)$ of Eq. (1.3), if one of the following conditions

$$
\begin{aligned}
& p=1, \quad \alpha<2 \beta, \quad b>\sigma ; \\
& 0<p<1, \quad \alpha=2 \beta, \quad b(1-p)>2|c|^{2}(\kappa+\bar{\kappa})
\end{aligned}
$$

is satisfied, then (3.2) holds. 
Applying Theorem 3.1 to Eq. (1.4), the condition (H1) should be replaced by $\left(\mathrm{H}^{\prime}\right)$, which is equivalent to choose $\bar{\kappa}=0$ in (H1). Then we may obtain the following corollary.

Corollary 3.3 Under the conditions (H1') and (H3), for the global positive solution $x(t)$ of Eq. (1.4), if one of the following conditions

$$
\begin{aligned}
& p=1, \quad \alpha<2 \beta, \quad b>\sigma ; \\
& 0<p<1, \quad \alpha=2 \beta, \quad b>\sigma+\frac{2|c|^{2} \kappa}{1-p}
\end{aligned}
$$

holds, then

$$
\limsup _{t \rightarrow \infty} \frac{\log \left(|x(t)|^{p}\right)}{\log (t)} \leq 1, \quad \text { a.s. }
$$

In these results above, the condition (H3) or ( $\left.\mathrm{H}^{\prime}\right)$ plays an important role to suppress growth of the solution. To use the condition $(\mathrm{H} 3)$ or $\left(\mathrm{H}^{\prime}\right)$, it is necessary to require $p \leq 1$. To avoid the condition ( $\mathrm{H} 3)$ or $\left(\mathrm{H}^{\prime}\right)$, we may impose another condition on the function $f$ to suppress growth of the solution. This idea leads to the following theorem.

Theorem 3.4 Under the conditions (H2) and (H4), for the global positive solution $x(t)$ of Eq. (1.2), if

$$
\alpha>2 \beta, \quad b>|c| \sigma / \hat{c}
$$

hold, then

$$
\limsup _{t \rightarrow \infty} \frac{\log (|x(t)|)}{\log (t)} \leq 0, \quad \text { a.s. }
$$

If $p \geq 1$ and

$$
\alpha=2 \beta, \quad b>\frac{|c| \sigma}{\hat{c}}+\frac{|c|(\lambda+\bar{\lambda})^{2}(p-1)}{2}
$$

hold, then

$$
\limsup _{t \rightarrow \infty} \frac{\log \left(|x(t)|^{p}\right)}{\log (t)} \leq 1, \quad \text { a.s. }
$$

Proof Repeating the proof process of Theorem 3.1 obtains Eq. (3.3). Then by the conditions $(\mathrm{H} 2)$ and $(\mathrm{H} 4)$, we may estimate $I_{1}$ and $I_{2}$. By the condition (H4),

$$
\begin{aligned}
I_{1} & \leq \frac{|\varphi(0)|^{-1}}{c^{T} \varphi(0)}\left[-b|\varphi(0)|^{\alpha}+\sigma \int_{-\infty}^{0}|\varphi(\theta)|^{\alpha} \mathrm{d} \bar{\mu}(\theta)+o\left(|\varphi(0)|^{\alpha}\right)\right] \\
& \leq-\frac{b}{|c|}|\varphi(0)|^{\alpha}+\frac{\sigma}{\hat{c}} \int_{-\infty}^{0}|\varphi(\theta)|^{\alpha} \mathrm{d} \bar{\mu}(\theta)+\mathrm{o}\left(|\varphi(0)|^{\alpha}\right) .
\end{aligned}
$$


Note the elementary inequality: for any $x, y \geq 0$ and $u \in(0,1)$

$$
(x+y)^{2} \leq \frac{x^{2}}{u}+\frac{y^{2}}{1-u}
$$

Then for any $u, v \in(0,1)$, by the condition (H2) and the Hölder inequality,

$$
\begin{aligned}
I_{2} & \leq \frac{(p-1)^{+}}{2}\left[\sum_{i=1}^{n} \frac{c_{i} \varphi_{i}(0)}{c^{T} \varphi(0)}\left|g_{i}(\varphi)\right|\right]^{2} \\
& \leq \frac{(p-1)^{+}}{2} \max _{1 \leq i \leq n}\left|g_{i}(\varphi(0))\right|^{2} \\
& \leq \frac{(p-1)^{+}}{2}\left[\lambda|\varphi(0)|^{\beta}+\bar{\lambda} \int_{-\infty}^{0}|\varphi(\theta)|^{\beta} \mathrm{d} v(\theta)+\mathrm{o}\left(|\varphi(0)|^{\beta}\right)\right]^{2} \\
& \leq \frac{(p-1)^{+}}{2 u}\left[\lambda|\varphi(0)|^{\beta}+\bar{\lambda} \int_{-\infty}^{0}|\varphi(\theta)|^{\beta} \mathrm{d} v(\theta)\right]^{2}+\mathrm{o}\left(|\varphi(0)|^{2 \beta}\right) \\
& \leq \frac{(p-1)^{+}}{2 u}\left[\frac{\lambda^{2}}{v}|\varphi(0)|^{2 \beta}+\frac{\bar{\lambda}^{2}}{1-v} \int_{-\infty}^{0}|\varphi(\theta)|^{2 \beta} \mathrm{d} v(\theta)\right]+\mathrm{o}\left(|\varphi(0)|^{2 \beta}\right) .
\end{aligned}
$$

Substituting $I_{1}$ and $I_{2}$ into (3.3) yields

$$
\begin{aligned}
\Phi_{\varepsilon} \leq & \frac{\sigma}{\hat{c}}\left[\int_{-\infty}^{0}|\varphi(\theta)|^{\alpha} \mathrm{d} \bar{\mu}(\theta)-\bar{\mu}_{\varepsilon}|\varphi(0)|^{\alpha}\right] \\
& +\frac{(p-1)^{+} \bar{\lambda}^{2}}{2 u(1-v)}\left[\int_{-\infty}^{0}|\varphi(\theta)|^{2 \beta} \mathrm{d} \nu(\theta)-v_{\varepsilon}|\varphi(0)|^{2 \beta}\right]+I,
\end{aligned}
$$

where

$$
I=-\left(\frac{b}{|c|}-\frac{\sigma \bar{\mu}_{\varepsilon}}{\hat{c}}\right)|\varphi(0)|^{\alpha}+\frac{(p-1)^{+}}{2 u}\left(\frac{\lambda^{2}}{v}+\frac{\bar{\lambda}^{2} v_{\varepsilon}}{1-v}\right)|\varphi(0)|^{2 \beta}+o\left(|\varphi(0)|^{\alpha}\right) .
$$

If the condition (3.8) holds, we can choose sufficiently small $\varepsilon$ such that $b>|c| \sigma \bar{\mu}_{\varepsilon} / \hat{c}>0$. Then, by Lemma 2.1, we have

$$
I=-\left(\frac{b}{|c|}-\frac{\sigma \bar{\mu}_{\varepsilon}}{\hat{c}}\right)|\varphi(0)|^{\alpha}+o\left(|\varphi(0)|^{\alpha}\right) \leq \text { const }
$$

Then, for any $p \geq 1$, Theorem 2.1 gives

$$
\limsup _{t \rightarrow \infty} \frac{\log (|x(t)|)}{\log t} \leq \frac{1}{p}, \quad \text { a.s. }
$$

Letting $p \rightarrow \infty$, we get the desired assertion (3.9).

If the condition (3.10) holds, noting $\alpha=2 \beta$ and $p \geq 1$, we have

$$
I=-\left[\frac{b}{|c|}-\frac{\sigma \bar{\mu}_{\varepsilon}}{\hat{c}}-\frac{(p-1)}{2 u}\left(\frac{\lambda^{2}}{v}+\frac{\bar{\lambda}^{2} v_{\varepsilon}}{1-v}\right)\right]|\varphi(0)|^{\alpha}+o\left(|\varphi(0)|^{\alpha}\right) .
$$


Assume that $\lambda, \bar{\lambda} \neq 0$ (when $\lambda, \bar{\lambda}=0$, the computation is obvious). Choose $v=\lambda /(\lambda+\bar{\lambda}) \in$ $(0,1)$ such that

$$
\bar{b}:=\frac{b}{|c|}-\frac{\sigma \bar{\mu}_{\varepsilon}}{\hat{c}}-\frac{(p-1)}{2 u}\left(\frac{\lambda^{2}}{v}+\frac{\bar{\lambda}^{2} v_{\varepsilon}}{1-v}\right)=\frac{b}{|c|}-\frac{\sigma \bar{\mu}_{\varepsilon}}{\hat{c}}-\frac{(p-1)}{2 u}(\lambda+\bar{\lambda})\left(\lambda+\bar{\lambda} v_{\varepsilon}\right) .
$$

Noting that the condition (3.10) holds, choose $\varepsilon$ sufficiently small and $u$ sufficiently near 1 such that $\bar{b}>0$. So $I \leq$ const. Applying Theorem 2.1 therefore gives the desired assertion (3.11).

The result (3.9) is a very strong result. It shows that growth of the solution $x(t)$ is so slow that it is near to be bound. By this result, we know that for any $\varepsilon>0$, there is a positive random time $T_{\varepsilon}$ such that, with probability one, for any $t \geq T_{\varepsilon}$,

$$
|x(t)| \leq t^{\varepsilon}
$$

In other words, with probability one, the solution will not grow fast than $t^{\varepsilon}$.

Applying Theorem 3.4 to Eq. (1.3), the condition (H2) should be replaced by $\left(\mathrm{H} 2{ }^{\prime}\right)$, which implies that $\bar{\lambda}=0$ in (H2). We may obtain the following corollary.

Corollary 3.5 Under the conditions $\left(H 2^{\prime}\right)$ and (H4), for the global positive solution $x(t)$ of Eq. (1.3), if

$$
\alpha>2 \beta, \quad b>|c| \sigma / \hat{c}
$$

then (3.9) holds. If $p \geq 1$ and

$$
\alpha=2 \beta, \quad b>\frac{|c| \sigma}{\hat{c}}+\frac{|c| \lambda^{2}(p-1)}{2}
$$

then (3.11) holds.

Applying Theorem 3.4 to Eq. (1.4), the condition (H4) should be replaced by ( $\left.\mathrm{H} 4^{\prime}\right)$, which implies that $\sigma=0$ in (H4). The corollary follows.

Corollary 3.6 Under the conditions (H2) and (H4'), for the global positive solution $x(t)$ of Eq. (1.4), if

$$
\alpha>2 \beta
$$

then (3.9) holds. If $p \geq 1$ and

$$
\alpha=2 \beta, \quad 2 b>|c|(\lambda+\bar{\lambda})^{2}(p-1),
$$

then (3.11) holds.

Comparing Theorem 3.1 with Theorem 3.4, we conclude that in Theorem 3.1, the function $g$ plays an important role to suppress growth of the solution in condition (H3). While in Theorem 3.4, the function $f$ is the main factor to suppress growth of the solution in condition (H4). 


\section{Some special case}

In this section, some special Kolmogorov-type equations are considered by applying the results in the previous two sections to obtain some pathwise estimation, where some special noises are chosen. We firstly consider the $n$-dimensional stochastic functional equations

$$
\mathrm{d} x(t)=\operatorname{diag}\left(x_{1}(t), \ldots, x_{n}(t)\right)\left[f\left(x_{t}\right) \mathrm{d} t+q \mathrm{~d} w(t)\right]
$$

which is equivalent to choosing $g(x) \equiv q \in \mathbb{R}^{n}$ in Eq. (1.2). Clearly, the condition (H2') is satisfied. Applying Corollary 3.5 to Eq. (4.1) gives the following theorem.

Theorem 4.1 Under the condition (H4), if $b>|c| \sigma / \hat{c}$, for the global positive solution $x(t)$ of Eq. (4.1), then the result (3.9) holds.

Then we consider the functional Lotka-Volterra-type system of Eq. (4.1)

$$
\mathrm{d} x(t)=\operatorname{diag}\left(x_{1}(t), \ldots, x_{n}(t)\right)\left[\left(a+A x(t)+B \int_{-\infty}^{0} x(t+\theta) \mathrm{d} \mu(\theta)\right) \mathrm{d} t+q \mathrm{~d} w(t)\right]
$$

where $a \in \mathbb{R}^{n}, A, B \in \mathbb{R}^{n \times n}$ and $\mu \in \mathcal{M}_{\varepsilon}$ for some suitable $\varepsilon \in\left(0, \varepsilon_{0}\right]$. For any $c=$ $\left(c_{1}, \ldots, c_{n}\right)^{T} \in \mathbb{R}_{++}^{n}$. Let $C=\operatorname{diag}\left(c_{1}, \ldots, c_{n}\right)$, Then we have

$$
\begin{aligned}
\varphi^{T}(0) C f(\varphi) & =\varphi^{T}(0) C\left[a+A \varphi(0)+B \int_{-\infty}^{0} \varphi(\theta) \mathrm{d} \mu(\theta)\right] \\
& \leq \varphi^{T}(0) C A \varphi(0)+\left|\varphi^{T}(0) C B\right| \int_{-\infty}^{0}|\varphi(\theta)| \mathrm{d} \mu(\theta)+\mathrm{o}\left(|\varphi(0)|^{2}\right) \\
& \leq \frac{1}{2} \lambda_{\max }^{+}\left(C A+A^{T} C\right)|\varphi(0)|^{2}+\check{c}|B||\varphi(0)| \int_{-\infty}^{0}|\varphi(\theta)| \mathrm{d} \mu(\theta)+\mathrm{o}\left(|\varphi(0)|^{2}\right) \\
& =:|\varphi(0)|\left[-b|\varphi(0)|+\sigma \int_{-\infty}^{0}|\varphi(\theta)| \mathrm{d} \mu(\theta)\right]+\mathrm{o}\left(|\varphi(0)|^{2}\right),
\end{aligned}
$$

where $2 b=-\lambda_{\max }^{+}\left(C A+A^{T} C\right), \sigma=\check{c}|B|$. Here we use the notation

$$
\lambda_{\max }^{+}(M)=\sup _{x \in \mathbb{R}_{+},|x|=1} x^{T} M x, \quad \text { for a symmetric matrix } M \in \mathbb{R}^{n \times n},
$$

which was introduced by Bahar and Mao [2]. Let us emphasize that this is different from the largest eigenvalue $\lambda_{\max }(M)$ of the matrix $M$. Recall the nice property of the largest eigenvalue:

$$
\lambda_{\max }(M)=\sup _{x \in \mathbb{R},|x|=1} x^{T} M x
$$

Clearly, $\lambda_{\max }^{+}(M) \leq \lambda_{\max }(M)$, which implies that $\lambda_{\max }^{+}(M)<0$ if the matrix $M$ is negative definite. (4.3) shows that $f$ satisfies the condition (H4) when we choose $\alpha=1$. The condition $b>|c| \sigma / \hat{c}$ is equivalent to

$$
-\lambda_{\max }^{+}\left(C A+A^{T} C\right)>2|c| \check{c}|B| / \hat{c}
$$

then Theorem 4.1 implies the following corollary. 
Corollary 4.2 If there exists $c=\left(c_{1}, \ldots, c_{n}\right)^{T} \in \mathbb{R}_{++}^{n}$ such that $C=\operatorname{diag}\left(c_{1}, \ldots, c_{n}\right)$ satisfies the condition (4.4), for the global positive solution $x(t)$ of Eq. (4.2) has the property (3.9).

If we choose $\mu$ is the Dirac measure at some point $0<\tau<\infty$, then Eq. (4.2) may be rewritten as

$$
\mathrm{d} x(t)=\operatorname{diag}\left(x_{1}(t), \ldots, x_{n}(t)\right)[(a+A x(t)+B x(t-\tau)) \mathrm{d} t+q \mathrm{~d} w(t)]
$$

which is another Lotka-Volterra type delay equation that has been investigated by Bahar et al. [2]. Applying Corollary 4.2 to Eq. (4.5) gives the following corollary.

Corollary 4.3 If there exists $c=\left(c_{1}, \ldots, c_{n}\right)^{T} \in \mathbb{R}_{++}^{n}$ such that $C=\operatorname{diag}\left(c_{1}, \ldots, c_{n}\right)$,

$$
-\lambda_{\max }^{+}\left(C A+A^{T} C\right)>2|B||c| \check{c} / \hat{c}
$$

then the global positive solution $x(t)$ of Eq. (4.5) has the property (3.9).

Now we consider another Kolmogorov-type equation

$$
\mathrm{d} x(t)=\operatorname{diag}\left(x_{1}, \ldots, x_{n}\right)\left[f\left(x_{t}\right) \mathrm{d} t+Q x(t) \mathrm{d} w(t)\right]
$$

where $Q=\left[q_{i j}\right] \in \mathbb{R}^{n \times n}$. Clearly, here $g$ satisfies the condition (H2') when we choose that $\lambda=|Q|$ and $\beta=1$. If the matrix $Q$ satisfies the condition

$$
q_{i i}>0 \quad \text { if } 1 \leq i \leq n, \quad \text { while } \quad q_{i j} \geq 0 \quad \text { if } i \neq j \text {, }
$$

then $g$ satisfies the condition $\left(\mathrm{H}^{\prime}\right)$ when we choose that $b=\max _{1 \leq i \leq n}\left\{c_{i}^{2} q_{i i}^{2}\right\}$. Then Corollary 3.2 gives the following theorem.

Theorem 4.4 Under the conditions (4.8) and (H1), for the global positive solutions of Eq. (4.7), if one of the following two conditions

$$
\begin{aligned}
& p=1, \quad \alpha<2 \\
& 0<p<1, \quad \alpha=2, \quad \max _{i \leq i \leq n}\left\{c_{i}^{2} q_{i i}^{2}\right\}>\frac{2|c|^{2}(\kappa+\bar{\kappa})}{1-p},
\end{aligned}
$$

is satisfied, where $c=\left(c_{1}, \ldots, c_{n}\right)^{T} \in \mathbb{R}_{++}^{n}$, then (3.2) holds.

Roughly speaking, Theorem 4.4 shows that under the condition (4.8), if the growth order $\alpha$ of $f$ is smaller than 2 (or $\alpha=2$ under some conditions), (3.2) is satisfied for all $p \leq 1$. To satisfy (3.2) under $p \leq 1$, the condition (4.8) plays a crucial role. But here $f$ is only required to satisfy an unrestrictive condition, which includes the linear growth condition. If $f$ is the linear growth function, namely,

$$
f(\varphi)=a+A \varphi(0)+B \int_{-\infty}^{0} \varphi(\theta) \mathrm{d} \mu(\theta)
$$


then Eq. (4.7) may be rewritten as

$$
\begin{aligned}
\mathrm{d} x(t)= & \operatorname{diag}\left(x_{1}(t), \ldots, x_{n}(t)\right) \\
& \times\left[\left(a+A x(t)+B \int_{-\infty}^{0} x(t+\theta) \mathrm{d} \mu(\theta)\right) \mathrm{d} t+Q x(t) \mathrm{d} w(t)\right],
\end{aligned}
$$

where $Q \in \mathbb{R}^{n \times n}$, which is another stochastic functional Lotka-Volterra systems. Clearly, $f$ satisfies the condition (H1) when we choose that $\alpha=1, \kappa=1, \bar{\kappa}=|B|$. So, for $p=1$, we have the following theorem.

Theorem 4.5 Under the condition (4.8), the global positive solution of Eq. (4.10) has the property

$$
\limsup _{t \rightarrow \infty} \frac{\log x(t)}{\log t} \leq 1, \quad \text { a.s. }
$$

We further choose that $\mu$ is a Dirac measure at the point $\tau$, then Eq. (4.10) is written as the delay stochastic Lotka-Volterra systems

$$
\mathrm{d} x(t)=\operatorname{diag}\left(x_{1}(t), \ldots, x_{n}(t)\right)[(a+A x(t)+B x(t-\tau)) \mathrm{d} t+Q x(t) \mathrm{d} w(t)],
$$

which is discussed by [1] where Theorem 5.3 is obtained. This means that our result is more general.

\section{Competing interests}

The authors declare that they have no competing interests.

\section{Authors' contributions}

The authors contributed equally and significantly in writing this article. The authors read and approved the final manuscript.

\section{Author details}

'School of Mathematics and Computing Sciences, Changsha University of Science and Technology, Changsha, Hunan

410076, China. ${ }^{2}$ School of Mathematics, Central South University, Changsha, Hunan 410075, China.

\section{Acknowledgements}

This work was supported in part by the National Natural Science Foundation of China under Grant nos. 11101054 11101434, the Open Fund Project of Key Research Institute of Philosophies and Social Sciences in Hunan Universities under Grant no. 11FEFM11, the Scientific Research Funds of Hunan Provincial Science and Technology Department of China under Grant no. 2012SK3096, and Hunan Provincial Natural Science Foundation of China under Grant no. 12 jj4005.

Received: 28 October 2011 Accepted: 20 July 2012 Published: 1 August 2012

\section{References}

1. Bahar, A, Mao, X: Stochastic delay Lotka-Volterra model. J. Math. Anal. Appl. 292, 364-380 (2004)

2. Bahar, A, Mao, X: Stochastic delay population dynamics. Int. J. Pure Appl. Math. 4, 377-400 (2004)

3. Tineo, A: Existence of global attractors for a class of periodic Kolmogorov systems. J. Math. Anal. Appl. 279, 365-371 (2003)

4. Granados, B: Permanence for a large class of planar periodic Kolmogorov systems. J. Math. Anal. Appl. 268, 701-706 (2002)

5. Tang, B, Kuang, Y: Permanence in Kolmogorov-type systems of nonautonomous functional differential equations. J. Math. Anal. Appl. 197, 427-447 (1996)

6. Chen, F: Global asymptotic stability in $n$-species non-autonomous Lotka-Volterra competitive systems with infinite delays and feedback control. Appl. Math. Comput. 170, 1452-1468 (2005)

7. $\mathrm{Wu}, \mathrm{F}, \mathrm{Hu}, \mathrm{S}$ : Stochastic functional Kolmogorov-type population dynamics. J. Math. Anal. Appl. 347, 534-549 (2008)

8. $\mathrm{Wu}, \mathrm{F}, \mathrm{Hu}, \mathrm{S}$ : On the pathwise growth rates of large fluctuations of stochastic population systems with innite delay. Stoch. Models 27, 94-119 (2011)

9. Wu, F, Hu, S, Liu, Y: Positive solution and its asymptotic behaviour of stochastic functional Kolmogorov-type system. J. Math. Anal. Appl. 364, 104-118 (2010) 
10. $\mathrm{Wu}, \mathrm{F}, \mathrm{Hu}, \mathrm{Y}$ : Pathwise estimation of the stochastic functional Kolmogorov-type system. J. Franklin Inst. 346, 191-205 (2009)

11. Bereketoglu, H, Gyori, I: Golbal asymptotic stability in a nonautonomous Lotka-Volterra type systems with infinite delay. J. Math. Anal. Appl. 210, 279-291 (1997)

12. Wang, J, Zhou, L, Tang, Y: Asymptotic periodicity of the Volterra equation with infinite delay. Nonlinear Anal. 68, 315-328 (2008)

13. Xu, R, Chaplain, MAJ, Chen, L: Global asymptotic stability in $n$-species nonautonmous Lotka-Volterra competitive systems with infinited delays. Appl. Math. Comput. 130, 295-309 (2002)

14. Zhou, S, Hu, S, Cen, L: Stochastic Kolmogorov-type system with infinite delay. Appl. Math. Comput. 218, 207-218 (2011)

15. Faria, T: Global attractivity in scalar delayed differential equations with applications to population models. J. Math Anal. Appl. 289, 35-54 (2004)

16. He, X, Gopalsamy, K: Persistence, attractivity, and delay in facultative mutualism. J. Math. Anal. Appl. 215, 154-173 (1997)

17. Mao, X, Marion, G, Renshaw, E: Environmental noise supresses explosion in population dynamics. Stoch. Process. Appl. 97, 96-110 (2002)

18. Mao, X, Sabanis, S, Renshaw, E: Asymptotic behaviour of the stochastic Lotka-Volterra model. J. Math. Anal. Appl. 287, $141-156(2003)$

19. Kuang, Y, Smith, HL: Global stability for infinite delay Lotka-Volterra type systems. J. Differ. Equ. 103, $221-246$ (1993)

20. Muroya, Y: Persistence and global stability in Lotka-Volterra delay differential systems. Appl. Math. Lett. 17, 759-800 (2004)

21. Teng, Z: The almost periodic Kolmogorov competitive systems. Nonlinear Anal. 42, 1221-1230 (2000)

doi:10.1186/1029-242X-2012-171

Cite this article as: Zhu and $\mathrm{Xu}$ : Pathwise estimation of stochastic functional Kolmogorov-type systems with infinite

delay. Journal of Inequalities and Applications 2012 2012:171.

\section{Submit your manuscript to a SpringerOpen ${ }^{\circ}$ journal and benefit from:}

- Convenient online submission

Rigorous peer review

- Immediate publication on acceptance

- Open access: articles freely available online

- High visibility within the field

- Retaining the copyright to your article 